\title{
ANALYSIS OF SHEAR LAG IN BOX BEAMS BY THE PRINCIPLE OF MINIMUM POTENTIAL ENERGY*
}

\author{
BY \\ ERIC REISSNER \\ .Jassachusetts Institute of Technology
}

1. Introduction. Let us consider a thin-walled box bean of web height $2 h$ and cover sheet width $2 w$ which is bent in such a way that one of the cover sheets is in tension while the opposite cover sheet is in compression (Fig. 1). In elementary beam theory the assumption is made that the normal stress in the cover sheets does not

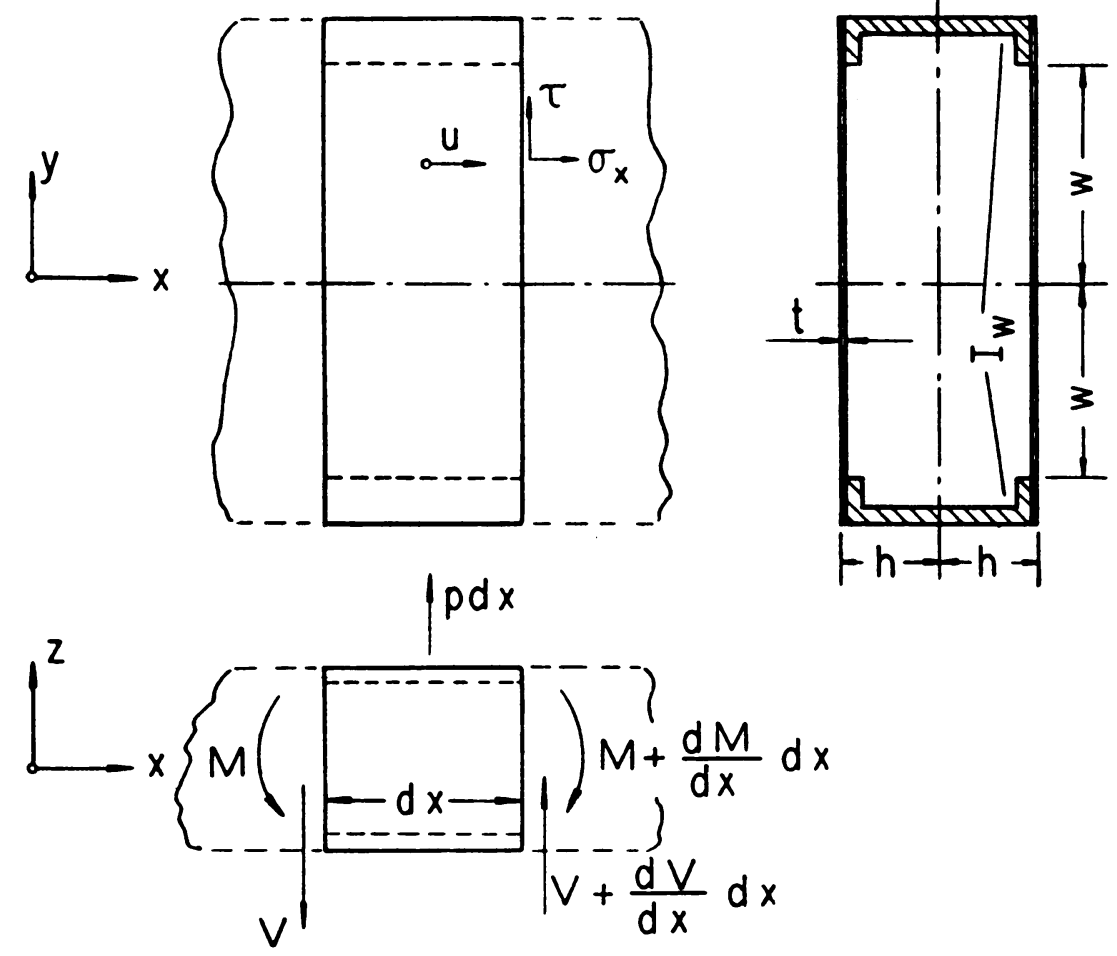

FIG. 1. Sketch of spanwise element of box beam with doubly symmetric cross section.

vary in the direction across the sheet. Because of the shear deformability of the cover sheets this assumption of elementary beam theory is of ten seriously in error for wide beams. In aeronautical engineering this effect is known under the name of shear lag. In recent papers, ${ }^{1,2}$ shear lag in box beams has been analyzed by an application

* Received Feb. 22, 1946.

${ }^{1}$ E. Reissner, Least work solutions of shear lag problems, Journal of the Aeronautical Sciences, 8, 284291 (1941). 
of the theorem of least work which is the basic minimum principle for the stresses. The present paper contains an application to the problem of shear lag of the theorem of minimum potential energy, which is the basic minimum principle for the strains. ${ }^{3}$ It is shown that application of the theorem of minimum potential energy to the present problem leads to simpler and more general results than the application of the theorem of least work. While the least-work method furnishes the stresses in box beams with no cut-outs, application of the minimum-potential-energy method furnishes, in a simpler manner, the stresses in beams without or with cut-outs. It also furnishes beam deflections, and is equally convenient for beams supported in statically determinate or in statically indeterminate manner.

Application, in the manner described below, of the minimum-potential-energy principle to the problem of bending of thin-walled box beams leads to a differential equation for the beam deflection which is a generalization of the relation $z^{\prime \prime}=-M / E I$; this differential equation contains an additional term proportional to the fourth derivative of $z$ which takes into account the shear deformability of the cover sheets. As the order of the differential equation in this theory is higher than the order of the differential equation of elementary beam theory, boundary conditions appear in addition to those of elementary beam theory. These additional boundary conditions are different for beams with cut outs and for beams without cut outs.

The manner of application of the results obtained in the present paper is shown by solving explicitly the following four examples.

1. Simply supported beam. Load distributed according to a cosine law.

2. Cantilever beam with uniform load distribution. Cover sheets fixed at the support.

3. Cantilever beam with uniform load distribution. Cover sheets not fixed at the support.

4. Beam with both ends built in. Uniform load distribution.

For the sake of simplicity, it is assumed in what follows that the cross sections of the beams are rectangular and doubly symmetrical. It also is assumed that there is no continuous variation of cross-sectional properties.

The author believes that the way in which the principle of minimum potential energy is here applied to the problem of shear lag will prove useful in other problems of structural mechanics. As an example of such future application, the theory for combined torsion and bending of beams with open or closed cross sections is mentioned.

2. Formulation and solution of problem. In the following, we analyze a box beam of doubly symmetrical rectangular cross section, composed of cover sheets, sidewebs and flanges. A given distribution of loads is applied to the sidewebs, acting normal to the plane of the cover sheets (Fig. 1). To this load distribution there corresponds a distribution of bending moments $M(x)$. The spanwise coordinate being $x$, let $y$ be the coordinate in the plane of the cover sheets perpendicular to the $x$ direction, and $z(x)$ the deflection of the neutral axis of the beam.

2F. B. Hildebrand and E. Reissner, Least work analysis of the problem of shear lag in box beams, N.A.C.A. Technical Note No. 893 (1943).

${ }^{3}$ For a formulation of these theorems see for instance I. S. Sokolnikoff and R. D. Specht, Mathematical theory of elasticity, McGraw-Hill·Book Co., Inc., New York, 1946; pp. 275-287. 
The potential energy of the bent beam may be considered as composed of three parts. The first part is the potential energy of the load system. This may be written in the form

$$
\Pi_{\iota}=\int M(x) \frac{d^{2} z}{d x^{2}} d x,
$$

the integral being extended over the entire length of the beam. ${ }^{4}$ The second part is the strain energy of sidewebs and flanges. This may be written in the form

$$
\mathrm{I}_{w}=\frac{1}{2} \int E I_{u}\left(\frac{d^{2} z}{d x^{2}}\right)^{2} d x,
$$

the quantity $I_{u}$ denoting the principal moment of inertia of the two sidewebs and flanges.

The third part is the strain energy of the two cover sheets. If it is assumed that the normal strains in the chordwise direction in the sheets may be neglected, as discussed in the reference given in Footnote 1, then the strain energy of the two sheets is given by the integral

$$
\mathrm{II}_{\mathrm{e}}=\frac{1}{2} \iint 2 \iota\left[\mathrm{E}_{x}^{2}+G \gamma^{2}\right] d x d y,
$$

where the quantity $t$ denotes the cover sheet thickness, and where $E$ and $G$ are the effective moduli of elasticity and rigidity. Spanwise normal strain $\epsilon_{x}$ and shear strain $\boldsymbol{\gamma}$ are then expressed in terms of the spanwise sheet displacement $u$ as follows

$$
\epsilon_{x}=\frac{\partial u}{\partial x}, \quad \gamma=\frac{\partial u}{\partial y} .
$$

The theorem of minimum potential energy states that the total potential energy

$$
\Pi=\Pi_{l}+\Pi_{w}+\Pi_{l}
$$

becomes a minimum for the correct displacement functions $u$ and $z$, if only such displacement functions are compared which satisfy all conditions of support and continuity imposed on the displacements.

Direct application of this condition by means of the calculus of variations leads to a partial differential equation for $u$ and to a complete system of boundary conditions. In what follows, an ordinary differential equation for the beam deflection $z$ and boundary conditions for it are obtained instead. This is done by making a suitable approximation for the sheet displacements $u$ and by applying the rules of the calculaof variations to the resultant approximate expression for the potential energy function.

A reasonable assumption for the spanwise sheet displacements is

$$
u(x, y)= \pm h\left[\frac{d z}{d x}+\left(1-\frac{y^{2}}{w^{2}}\right) U(x)\right] .
$$

4 Eq. (1) implies that the beam is supported in such a manner that the end forces and moments can do no work. This restriction shortens the developments slightly. 
The second term on the right of F.q. (6) represents the correction due to shear lag. Instead of the vanishing chordwise variation of the sheet displacements of elementary beam theory, we now assume a parabolic variation. The relative magnitude of the function $U$ is a measure for the magnitude of the shear lag effect. The form of the correction is such that continuity of the displacements along the flanges, that is along $y= \pm w$, is preserved.

Denoting differentiation with respect to $x$ by primes, we obtain the following expressions for the strains in the sheets from Eqs. (6) and (4):

$$
\begin{aligned}
& \epsilon_{x}= \pm h\left[z^{\prime \prime}+\left(1-\frac{y^{2}}{w^{2}}\right) U^{\prime}\right], \\
& \gamma=\mp \frac{2 h}{w} \frac{y}{w} U .
\end{aligned}
$$

On the basis of Eqs. (7) and (8) the following expression for the strain energy of the sheets is obtained:

$$
\Pi_{s}=\iint t h^{2}\left\{E\left[z^{\prime \prime}+\left(1-\frac{y^{2}}{w^{2}}\right) U^{\prime}\right]^{2}+G\left[\frac{2}{w} \frac{y}{w} U\right]^{2}\right\} d y d x .
$$

In Eq. (9) the integration with respect to $y$ is carried out. Setting

$$
I_{s}=4 w h^{2}, \quad I=I_{s}+I_{w},
$$

we have

$$
\Pi_{s}=\frac{1}{2} \int E I_{\cdot}\left\{\left(z^{\prime \prime}\right)^{2}+\frac{8}{15}\left(U^{\prime}\right)^{2}+\frac{4}{3} z^{\prime \prime} U^{\prime \prime}+\frac{G}{E} \frac{4}{3 w^{2}} U^{2}\right\} d x .
$$

Substituting Eqs. (11), (12) and (1) into Eq. (5), we obtain the following expression for the potential energy of the system

$$
\begin{aligned}
\Pi= & \int\left\{\frac{1}{2} E I\left(z^{\prime \prime}\right)^{2}+M z^{\prime \prime}\right\} d x \\
& +\int \frac{1}{2} E I_{s}\left\{\frac{8}{15}\left(U^{\prime}\right)^{2}+\frac{4}{3} z^{\prime \prime} U^{\prime}+\frac{G}{E} \frac{4}{3 w^{2}} U^{2}\right\} d x .
\end{aligned}
$$

Differential equations and boundary conditions for $z$ and $U$ are obtained by making

$$
\delta \Pi=0 .
$$

Thus, with $x_{1}$ and $x_{2}$ denoting the ends of the interval of integration,

$$
\begin{aligned}
\delta \Pi= & \int\left\{\left[E I z^{\prime \prime}+M+\frac{2}{3} E I_{s} U^{\prime}\right] \delta z^{\prime \prime}\right. \\
& \left.+E I_{s}\left[-\frac{8}{15} U^{\prime \prime}-\frac{2}{3} z^{\prime \prime \prime}+\frac{G}{E} \frac{4}{3 w^{2}} V\right] \delta C^{\prime}\right\} d x \\
& +\left\{E I_{s}\left[\frac{8}{15} U^{\prime}+\frac{2}{3} z^{\prime \prime}\right] \delta U\right\}_{x_{1}}^{x_{2}}=0 .
\end{aligned}
$$


As $\delta z^{\prime \prime}$ and $\delta U$ are arbitrary in the interior of the interval $\left(x_{1}, x_{2}\right)$ the terms multiplying them must vanish. This gives the following two differential equations

$$
\begin{gathered}
z^{\prime \prime}+\frac{2}{3} \frac{I_{s}}{I} U^{\prime}+\frac{M}{E I}=0, \\
E I_{s}\left[l^{\prime \prime}-\frac{5}{2} \frac{G}{E} \frac{l^{\prime}}{w^{2}}+\frac{5}{4} z^{\prime \prime \prime}\right]=0 .
\end{gathered}
$$

The integrated portion of Eq. (14) defines the boundary and transition conditions for the function $U$. At a section where the sheet is fixed, $\delta U=0$ and

$$
t=0 \text {. }
$$

At a section where the sheet is not fixed and consequently $\delta U$ is arbitrary,

$$
E I_{s}\left[C^{\prime \prime}+\frac{s}{4} z^{\prime \prime}\right]=0 .
$$

Transitions conditions for adjacent bays with different stiffness are:

$$
U \text { and } E I_{0}\left[U^{\prime}+\frac{5}{4} z^{\prime \prime}\right] \text { continuous. }
$$

The above boundary and transition conditions are in addition to those imposed on $z$ and $M$ in elementary beam theory, as may be verified by repeated integration by parts of the term containing $\delta z^{\prime \prime}$ in the integral of Eq. (14).

3. The modified beam equation and its boundary conditions. By eliminating the quantity $U$ from Eqs. (15) to (19), we obtain a system of relations containing the beam deflection $z$ only.

The differential equation for $z$ is derived by differentiating Eq. (16) and substituting $U^{\prime}$ from Eq. (15). There follows

$$
z^{\prime \prime}+\frac{M}{E I}-w^{2} \frac{E}{G}\left[\frac{2}{5}\left(z^{\prime \prime}+\frac{M}{E I}\right)^{\prime \prime}-\frac{I_{s}}{3 I} z^{\mathrm{IV}}\right]=0 .
$$

When the shear deformability of the sheets is neglected, that is when it is assumed that $G=\infty$, Eq. (20) reduces to the well known result of elementary beam theory.

Equation (20) may be written in the alternate form

$$
z^{\prime \prime}-\frac{2}{5} \frac{E}{G}\left(1-\frac{5}{6} \frac{I_{6}}{I}\right) \frac{z^{\mathrm{IV}}}{w^{2}}=-\frac{M}{E I}+\frac{2}{5} \frac{E}{G} \frac{M^{\prime \prime}}{w^{2} E I} .
$$

With the help of Eqs. (15) and (16), the boundary condition (17), which holds when the sheet is attached to the support, is transformed into

$$
\left(1-\frac{5}{6} \frac{I_{0}}{I}\right) z^{\prime \prime \prime}+\frac{M^{\prime}}{E I}=0 \text {. }
$$

Similarly, the boundary condition (18), which holds when the sheet is not attached to the support, becomes

$$
\left(1-\frac{5}{6} \frac{I_{0}}{I}\right) z^{\prime \prime}+\frac{M}{E I}=0 .
$$


The continuity conditions (19) may be transformed in an analogous manner.

The values of the sheet stresses may be obtained from Eqs. (9) and (10). From Eq. (9) it follows that the flange stress is given by

$$
\sigma_{f}= \pm E h z^{\prime \prime} .
$$

For the application of the results it may be noted that the differential equation (21) can first be solved for the value of $z^{\prime \prime}$ which, according to (24), gives directly the approximate value of the flange stress $\sigma_{f}$. The magnitude of the deflection $z$ can then be found from the value of $z^{\prime \prime}$ as in elementary beam theory.

For the evaluation of the solution we define the following two parameters

$$
\begin{aligned}
& n=\frac{1}{1-5 I_{s} / 6 I}, \\
& k=\frac{1}{w} \sqrt{\frac{5 n}{2} \frac{G}{E}} .
\end{aligned}
$$

With (25) and (26) the differential equation (21) becomes

$$
z^{\prime \prime}-\frac{1}{k^{2}} z^{\mathrm{IV}}=-\frac{M}{E I}+\frac{n}{k^{2}} \frac{M^{\prime \prime}}{E I}
$$

the boundary condition at an end section where the sheet is attached to the support becomes

$$
z^{\prime \prime \prime}=-n \frac{M^{\prime}}{E I}
$$

and the boundary condition at an end section where the sheet is not attached to the support becomes

$$
z^{\prime \prime}=-n \frac{M}{E I} .
$$

4. Examples of applications (Fig. 2). 1. Simply supported beam. Load distributed according to a cosine law. Designating the span length of the beam by $l$ and assuming the origin of the coordinate system at the center of the beam we consider the moment distribution

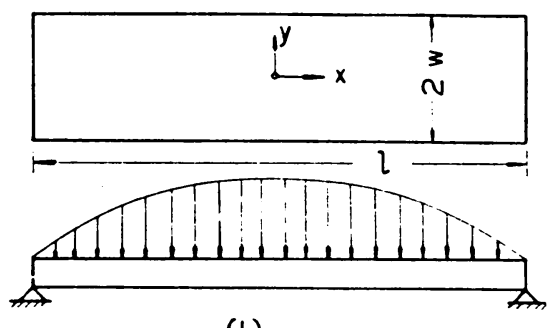

(I)

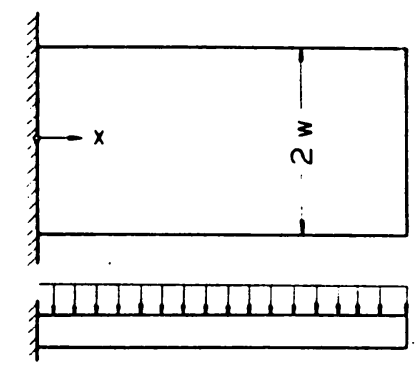

(2)

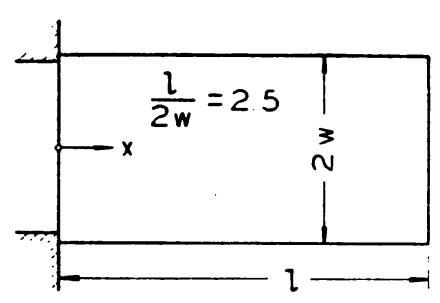

(3)
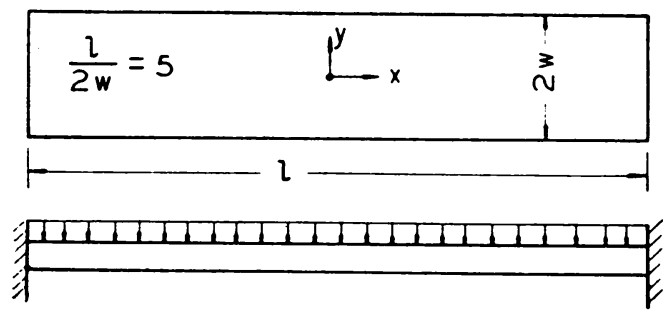

(4)

Fig. 2. Diagrammatic sketches of beams analyzed as examples of application of the theory. 


$$
M=M_{0} \cos \pi \frac{x}{l} .
$$

A particular solution of Eq. (27) is

$$
z=\left(\frac{l}{\pi}\right)^{2} \frac{M_{0}}{E I} \frac{1+n(\pi / k l)^{2}}{1+(\pi / k l)^{2}} \cos \pi \frac{x}{l} .
$$

As Eq. (31) satisfies the boundary condition (29) and the condition of vanishing deflection at the ends of the beam, it is the complete expression for the deflection function. When $1 / k=0, \mathrm{Eq}$. (31) reduces to the expression for $z$ in the case where shear lag is not taken into account. The factor

$$
\frac{1+n(\pi / k l)^{2}}{1+(\pi / k l)^{2}}=\frac{1+\left(2 \pi^{2} E / 5 G\right)(w / l)^{2}}{1+\left(2 \pi^{2} E / 5 G\right)(w / l)^{2}\left(1-5 I_{s} / 6 I\right)} .
$$

expresses the effect of shear lag on deflection and flange stresses.

2. Cantilever beam with uniform load distribution. Cover sheets fixed at support. Assuming that, contrary to what is indicated in Fig. 2, the free end of the beam has the coordinate $x=0$ and the fixed end of the beam the coordinate $x=l$, we may write the moment distribution in the form

$$
M=M_{0}\left(\frac{x}{l}\right)^{2}
$$

The differential equation (27) then becomes

$$
z^{\prime \prime}-\frac{1}{k^{2}} z^{1 \mathrm{~V}}=-\frac{M_{0}}{E I}\left[\left(\frac{x}{l}\right)^{2}-\frac{2 n}{(k l)^{2}}\right] .
$$

Solving for $z^{\prime \prime}$, we find

$$
z^{\prime \prime}=\frac{M_{0}}{E I}\left\{C_{1} \sinh k x+C_{2} \cosh k x-\left(\frac{x}{l}\right)^{2}+\frac{2(n-1)}{(k l)^{2}}\right\} .
$$

Satisfying the boundary condition (29) when $x=0$ and (28) when $x=l$, we obtain

$$
z^{\prime \prime}=-\frac{M_{0}}{E I}\left\{\left(\frac{x}{l}\right)^{2}+\frac{2(n-1)}{(k l)^{2}}\left[(\cosh k x-1)-\frac{\sinh k l-k l}{\cosh k l} \sinh k x\right]\right\} .
$$

According to Eq. (24), the flange stress at the fixed end of the beam becomes

$$
\sigma_{f}(l)=\mp \frac{M_{0} h}{I}\left\{1+\frac{2(n-1)}{k l}\left[\tanh k l-\frac{1}{k l}+\frac{1}{k l \cosh k l}\right]\right\} .
$$

We take for a numerical example

$$
\frac{I^{\circ}}{I}=\frac{1}{2}, \quad \frac{G}{E}=\frac{3}{8}, \quad \frac{l}{2 w}=\frac{5}{2},
$$

so that according to Eqș. (25) and (26)

$$
n^{-}=1.714, \quad k l=6.34,
$$


and we find

$$
\sigma_{f}(l)=\mp \frac{M_{0} h}{I}\{1+.190\} \text {. }
$$

By application of the least work method ${ }^{1,2}$ a factor 1.186 is obtained instead of the factor 1.190 in Eq. (40).

The deflection of the beam is obtained from Eq. (36) by integrating twice and making $z(l)=z^{\prime}(l)=0$. In the present case, the correction due to shear lag for the maximum deflection is about ten percent.

3. Cantilever beam with uniform load distribution. Cover sheets not fixed at support. Moment distribution and differential equation are given by Eqs. (33) and (34). The constants of integration in (36) are determined by satisfying Eq. (29) for $x=0$ and for $x=l$. There follows

$$
\begin{aligned}
z^{\prime \prime}=-\frac{M_{0}}{E I}\left\{\left(\frac{x}{l}\right)^{2}+\frac{2(n-1)}{(k l)^{2}}[(\cosh k x-1)\right. & \left.\left.-\frac{\cosh k l-1-\frac{1}{2}(k l)^{2}}{\sinh k l} \sinh k x\right]\right\} .
\end{aligned}
$$

Taking again $I_{s} / I=.5$, we should have, for the flange stress at the supported end, a value twice as large as the stress according to elementary beam theory for a beam with sheet attached to the support. In the present solution the factor 2 is replaced by $n=1.714$. This indicates that with the assumed parabolic chordwise variation of sheet displacement the condition that at the support of the beam the sheet is free of stress is only approximately satisfied. The same difficulty arises in methods which incorporrate the ability of the sheet to carry normal stresses as effective width contributions to the strength of stiffners. ${ }^{5}$ This difficulty is not serious when the main purpose of such "cut-out" calculations is the determination of the distance over which the cutout is effective and its effect on the over all beam stiffness. ${ }^{6}$

The localization of the effect of the cut-out may be seen by writing (41) in the form

$$
z^{\prime \prime} \approx-\frac{M_{0}}{E I}\left\{\left(\frac{x}{l}\right)^{2}+(n-1) e^{-k l(1-x / l)}\right\} .
$$

This equation indicates that the influence of the cut-out is small as soon as the distance $l-x$ satisfies the inequality

$$
l-x \gg \frac{-\log (n-1)}{k}=w \sqrt{\frac{2 E}{5 G}\left(1-\frac{5}{6} \frac{I_{s}}{I}\right)} \log \left(\frac{6}{5} \frac{I}{I_{s}}-1\right) .
$$

Thus, the wider the sheet and the smaller the value of the shear modulus $G$, the farther away does the effect of the cut-out extend in the spanwise direction.

The magnitude of the beam deflection is obtained from (41) in the form

\footnotetext{
5 P. Kuhn and P. Chiarito, Shear lag in box beams-methods of analysis and experimental investigations, N.A.C.A. Technical Report No. 739 (1943).

${ }^{6}$ Exact solutions of problems of this kind have been obtained by F. B. Hildebrand, The exact solution of shear-lag problems in flat panels and box beams assumed rigid in the transverse direction, N.A.C.A., Technical Note No. 894 (1943).
} 


$$
z(x)=\int_{1}^{x} \int_{1}^{x_{1}} \tilde{s}^{\prime \prime}\left(x_{2}\right) d x_{2} d x_{1}
$$

which determines the constants of integration such that $z(l)=z^{\prime}(l)=0$. For the deflection at the free end of the beam, we have

$$
z(0)=\frac{M_{0} l^{2}}{E I}\left\{\frac{1}{4}+\frac{2(n-1)}{k l}\left[\left(\frac{1}{2}+\frac{1}{(k l)^{2}}\right) \operatorname{coth} k l-\frac{1}{k l}-\frac{1}{(k l)^{2} \sinh k l}\right]\right\} .
$$

For a beam with dimensions as in (38) and (39), Eq. (45) becomes

$$
s(0)=\frac{M_{0} l^{2}}{E I}(.25+.083) \text {. }
$$

This indicates that for a beam with dimensions as given shear lag due to lack of sheet restraint at the supported end of the beam is responsible for a thirty percent increase of the maximum beam deflection as compared with the result of elementary beam theory for a beam fully restrained at the supported end. This increase of deflection of thirty percent compares with one of hundred per cent which is obtained if the contribution of the cover sheets is neglected.

4. Beam with both ends built-in. Uniform load distribution. The distribution of bending moments may be written as

$$
M=M_{0}\left(\frac{x}{l}\right)^{2}+M_{1}
$$

The value of $M_{0}$ is determined by the load intensity, the value of $M_{1}$ in this statically indeterminate problem has to be determined from the displacement boundary conditions. The boundary conditions are

$$
\begin{array}{r}
z\left( \pm \frac{l}{2}\right)=0, \quad \text { (48) } \quad z^{\prime}\left( \pm \frac{l}{2}\right) \\
z^{\prime \prime \prime}\left( \pm \frac{l}{2}\right)=-n \frac{M^{\prime}( \pm l / 2)}{E I} .
\end{array}
$$

For these boundary conditions the moment distribution is not affected by shear lag, provided the moment distribution is symmetrical about the mid-span section of the beam. Indeed, the differential equation (27) may be integrated to give

$$
z^{\prime}-\frac{z^{\prime \prime \prime}}{k^{2}}=-\int_{0}^{x} \frac{M}{E I} d x+\frac{n}{k^{2}} \frac{M^{\prime}}{E I}
$$

the limits of integration being so chosen that Eq. (51) satisfies the conditions of zero slope and zero vertical shear at the mid-span section. In view of (49) and (50), Eq. (51) implies

$$
\int_{0}^{l / 2} \frac{M}{E I} d x=0
$$


regardless of whether or not shear lag is taken into account. A considerably less simple proof of the same fact by means of the least work method has been given in the reference quoted in Footnote 2. For the moment distribution of Eq. (47) there follows, from (52),

$$
\frac{M_{0}}{24}+\frac{M_{1}}{2}=0,
$$

and hence

$$
M=M_{0}\left[\left(\frac{x}{l}\right)^{2}-\frac{1}{12}\right] .
$$

With this value of $M$ and the requirement that $z^{\prime \prime}$ be an even function of $x$, Eq. (27) is solved in the form

$$
z^{\prime \prime}=-\frac{M_{0}}{E I}\left\{\left(\frac{x}{l}\right)^{2}-\frac{1}{12}-\frac{2(n-1)}{(k l)^{2}}+C_{2} \cosh k x\right\} .
$$

The constant $C_{2}$ is determined from Eq. (50). There follows,

$$
z^{\prime \prime}=-\frac{M_{0}}{E}\left\{\left(\frac{x}{l}\right)^{2}-\frac{1}{12}+\frac{n-1}{k l}\left[\frac{\cosh k x}{\sinh k l / 2}-\frac{1}{k l / 2}\right]\right\} .
$$

Taking a beam five times as long as wide, that is $l / 2 w=5$, and assuming the remaining parameters as in (38) and (39), we obtain the following expressions for the flange stresses at the built-in section and at the center section of the beam

$$
\begin{aligned}
\sigma_{f}\left(\frac{l}{2}\right) & = \pm \frac{M_{0} h}{I} \frac{1}{6}(1+.283), \\
\sigma_{f}(0) & =\mp \frac{M_{0} h}{I} \frac{1}{12}(1+.106) .
\end{aligned}
$$

These results agree to within a fraction of a percent with the corresponding results obtained by the least work method. ${ }^{2}$ It is worthy of note that, for this beam with both ends built-in, shear lag is considerably larger than for a cantilever beam with the same load, same width and half the span of the beam with both ends built-in. If both beams had the same span, the discrepancy would be even larger.

The deflection $z$ of the beam is obtained from (56) and (48) in the form

$$
\begin{aligned}
& z=-\frac{M_{0} l^{2}}{E I}\left\{\frac{1}{12}\left(\frac{x}{l}\right)^{4}-\frac{1}{24}\left(\frac{x}{l}\right)^{2}+\frac{1}{192}\right.
\end{aligned}
$$

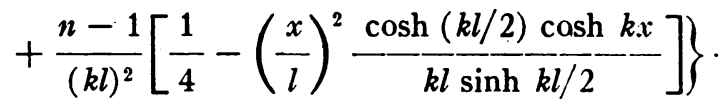

Corresponding to the stresses of Eqs. (57) and (58) we find for the deflection at midspan

$$
z(0)=-\frac{1}{192} \frac{M_{0} l^{2}}{E I^{-}}(1+.145)
$$


Shear lag in this beam is thus responsible for an almost fifteen percent increase in deflection. This percentage increase of deflection, while appreciable, is considerably smaller than the percentage increase of maximum flange stress.

Acknowledgment. The results of this paper were obtained in 1944 as part of work done for the structures department of the Research Laboratory of the Curtiss-Wright Corporation (now Cornell Aeronautical Laboratory). For permission to publish this paper the author is indebted to A. F. Donovan, Chief of the structures department of the Laboratory. 